\title{
Association between LMP2 and LMP7 gene polymorphisms and the risk of gastric cancer: A case-control study
}

\author{
XIANG MA $^{1 *}$, CHAO YANG $^{1 *}$, RAN TANG $^{1 *}$, ZEKUAN XU $^{1}$, ZHIHONG ZHANG ${ }^{2}$, \\ YOUNAN WANG ${ }^{1}$, JINGJING ZHANG ${ }^{1,3}$ and LI YANG $^{1}$ \\ Departments of ${ }^{1}$ General Surgery and ${ }^{2}$ Pathology, The First Affiliated Hospital of Nanjing Medical University; \\ ${ }^{3}$ Jiangsu Province Academy of Clinical Medicine, Institute of Tumor Biology, Nanjing, Jiangsu 210029, P.R. China
}

Received June 23, 2014; Accepted April 8, 2015

DOI: $10.3892 / 01.2015 .3154$

\begin{abstract}
The integrality of low molecular weight protein (LMP)2/LMP7 function plays an important role in the processing of GC cell antigens. The purpose of the present hospital-based case-control study was to estimate the effect of polymorphisms in the LMP2 and LMP7 genes on the risk of GC. Polymerase chain reaction-restriction fragment length polymorphism analysis was used to distinguish the Arg to His substitution at codon 60 of LMP2 (LMP2-60) and the Gln to Lys substitution at codon 145 of LMP7 (LMP7-145) in 502 gastric cancer patients and 502 age and gender-matched cancer-free control individuals. The Lys allele of the LMP7-145 variant was more frequent in GC patients compared with control individuals $[\mathrm{P}=0.004$; adjusted odds ratio (OR), 1.39; 95\% confidence interval (CI), 1.11-1.74]. The Gln/Lys and Lys/Lys genotypes increased the risk of GC compared with the Gln/Gln genotype $(\mathrm{P}=0.049$ and $\mathrm{P}=0.041$, respectively; adjusted OR, 1.32 and 2.13, respectively; $95 \%$ CI, 1.00-1.73 and 1.03-4.39, respectively). Compared with the Gln/Gln genotype, the LMP7-145 Gln/Lys and Lys/Lys variants of the LMP7 gene were also associated with increased susceptibility to $\mathrm{GC}(\mathrm{P}=0.017$; adjusted $\mathrm{OR}, 1.38$; $95 \% \mathrm{CI}, 1.06-1.80)$. Haplotype analysis revealed that the LMP2 (Arg)-LMP7 (Lys) haplotype was associated with increased risk of $\mathrm{GC}(\mathrm{P}=0.013$, adjusted $\mathrm{OR}=1.34,95 \% \mathrm{CI}=1.06-1.70)$. Stratified analysis revealed that the association between the risk of GC and the variant genotypes of LMP7-145 was stronger in older individuals (>59 years), males and non-smokers. However, no association between the LMP2-60 polymorphism and the risk of GC was observed. The present results suggest that the LMP7-145 genetic variant contributes to increased susceptibility to GC, and the Lys allele is an independent risk factor for GC.
\end{abstract}

Correspondence to: Dr Li Yang, Department of General Surgery, The First Affiliated Hospital of Nanjing Medical University, 300 Guangzhou Road, Nanjing, Jiangsu 210029, P.R. China

E-mail: pwkyangli@163.com

${ }^{*}$ Contributed equally

Key words: gastric cancer, LMP2/LMP7, gene polymorphism

\section{Introduction}

Gastric cancer (GC) remains the second leading cause of cancer-associated mortality worldwide $(1,2)$, and approximately one-half of worldwide gastric cancer cases occur in China (3). Similar to other malignancies, the mechanism of gastric carcinogenesis involves a complex multi-stage and multi-factorial process that depends on gene-environment interactions (4). In previous epidemiological studies, genetic factors were demonstrated to play an important role in the development of gastric cancer (5-8).

The expression of human leukocyte antigen (HLA) class I antigens on the surface of tumor cells is critical for the recognition of these cells by antigen-specific cytotoxic T lymphocytes (CTLs), not only for the initiation of CTL-associated inflammation but also for the anticancer immune response. An HLA-class I restricted immune response has been reported to eliminate GC cells, and the expression of HLA-class I antigens may be essential for the host immune response against cancer (9). Furthermore, downregulation of the expression of the HLA-class I antigen in GC is associated with tumor progression and tumor histology (10). Therefore, HLA-class I antigens may play a crucial role in the development and progression of GC.

Several steps lead to the generation of the tumor antigen-derived peptides that are presented by HLA class I antigens to cognate CTLs. In particular, tumor antigens are degraded into short peptides by the proteasome and immunoproteasome subunits low molecular weight protein (LMP) 2 and LMP7. The resulting peptides are transported into the endoplasmic reticulum (ER) lumen by transporter associated with antigen presentation (TAP). In the ER, newly synthesized HLA class I heavy chains assemble with $\beta 2$-microglobulin and peptides. Upon peptide binding, the HLA class I heterotrimeric complex is released from the ER and transported to the cell surface via the constitutive secretory pathway $(11,12)$. Therefore, the integrality of LMP2 and LMP7 function plays an important role in the processing of GC cell antigens. The present study hypothesized that the processing of gastric tumor antigens may be influenced by structural differences encoded by the LMP alleles.

Several studies have reported an association between the genetic variants of Arg to His substitution at codon 60 of 
Table I. Demographic characteristics of patients with gastric cancer and control individuals $(\mathrm{n}=502)$.

\begin{tabular}{|c|c|c|c|}
\hline \multirow[b]{2}{*}{ Characteristics } & \multicolumn{2}{|c|}{ Value per group, $\mathrm{n}(\%)$} & \multirow[b]{2}{*}{ P-value } \\
\hline & Gastric cancer & Control & \\
\hline Gender & & & 0.188 \\
\hline Male & $364(72.5)$ & 345 (68.7) & \\
\hline Female & $138(27.5)$ & $157(31.3)$ & \\
\hline $\begin{array}{l}\text { Median age, } \\
\text { years (25th-75th percentiles) }\end{array}$ & $60(52-66)$ & $59(49-69)$ & 0.916 \\
\hline Hypertension & & & 0.439 \\
\hline Yes & $129(25.7)$ & $140(27.9)$ & \\
\hline No & $373(74.3)$ & $362(72.1)$ & \\
\hline Diabetes & & & 0.236 \\
\hline Yes & $52(10.4)$ & $64(12.7)$ & \\
\hline No & $450(89.6)$ & $438(87.3)$ & \\
\hline Smoking & & & 0.003 \\
\hline Smoker & $123(24.5)$ & $85(16.9)$ & \\
\hline Non-smoker & $379(75.5)$ & $417(83.1)$ & \\
\hline Residence & & & 0.200 \\
\hline Rural & $289(57.6)$ & $265(52.8)$ & \\
\hline Urban & $213(42.4)$ & $237(47.2)$ & \\
\hline
\end{tabular}

LMP2 (LMP2-60) and Gln to Lys substitution at codon 145 of LMP7 (LMP7-145) and the risk of numerous malignant diseases, including ovarian cancer, colon cancer and esophageal carcinoma (13-15). However, the association between LMP2 and LMP7 polymorphisms and the risk of GC has not been investigated to date. The present study evaluated this association by conducting a hospital-based case-control study.

\section{Materials and methods}

Ethical approval and patient consent. The present study was approved by the Ethics Committee of the First Affiliated Hospital of Nanjing Medical University (Nanjing, Jiangsu, China). Written informed consent was obtained from each subject prior to enrollment in the present study.

Subjects. The present hospital-based case-control study included 502 GC cases and 502 cancer-free controls. All participants were genetically unrelated and resided in Jiangsu or the surrounding regions. All GC patients were consecutively recruited at the First Affiliated Hospital of Nanjing Medical University between May 2010 and April 2014. The diagnosis of GC was confirmed by endoscopic biopsy or surgical specimens in all patients. The cancer-free control subjects were matched with the patients by gender and possessed an age difference of less than five years. The control subjects were randomly selected from the First Affiliated Hospital of Nanjing Medical University during the same selection period as the patients with GC. Subjects with secondary recurrent malignancies, genetic diseases or those who underwent non-self blood transfusions were excluded from the present study, as were patients that received chemotherapy or radiotherapy. The data on the age, gender, smoking status, urban or rural residence, hypertension, diabetes and personal medical history of the patients were collected by questionnaire or medical records. Individuals that formerly or continued to smoke $\geq 10$ cigarettes per day for at least two years were defined as smokers. Former smokers that had successfully stopped smoking for $>2$ years were defined as non-smokers.

Genotyping and haplotype construction. Genomic DNA was extracted from $2 \mathrm{ml}$ of peripheral blood using standard methods, and the protocol used for the genomic DNA extraction was described in a previous study (16). LMP2 and LMP7 gene polymorphisms were genotyped using the polymerase chain reaction-restriction fragment length polymorphism (PCR-RFLP) assay. PCR reactions were performed in a total volume of $20 \mu \mathrm{l}$ of reaction mixtures containing $2 \mu 1$ 10X PCR buffer (Fermentas, Waltham, MA, USA), $1.75 \mathrm{mmol} / 1 \mathrm{MgCl}_{2}, 0.15 \mathrm{mmol} / 1 \mathrm{dNTP}, 1$ unit Taq polymerase (Fermentas), $150 \mathrm{ng}$ genomic DNA and $0.25 \mu \mathrm{mol} / 1$ of each primer. The primers used were as follows: LMP2-60 forward, 5'-CTCCACTTTACAGATGCAGA-3' and reverse, 5'-ACT TGGTGACTGTTGACTCC-3'; and LMP7-145 forward, 5'-TCATGGCGCTACTAGATGTATG-3' and reverse, 5'-AAC TCTTTGTCCTAACTTGCAC-3'. For PCR amplification, an initial denaturation was performed at $95^{\circ} \mathrm{C}$ for $2 \mathrm{~min}$, followed by 30 cycles of denaturation at $95^{\circ} \mathrm{C}$ for $35 \mathrm{sec}$, annealing at $56.5^{\circ} \mathrm{C}$ for LMP2-60, or $57.5^{\circ} \mathrm{C}$ for LMP7-145, for $30 \mathrm{sec}$ and elongation at $72^{\circ} \mathrm{C}$ for $45 \mathrm{sec}$, with a final elongation step at $72^{\circ} \mathrm{C}$ for $10 \mathrm{~min}$.

For RFLP, the 330-bp and 351-bp PCR products for the LMP2-60 and LMP7-145 polymorphisms were digested using 
Table II. Association between polymorphisms in the LMP2 and LMP7 genes and the risk of gastric cancer.

\begin{tabular}{|c|c|c|c|c|c|c|}
\hline \multirow[b]{2}{*}{ Genotype } & \multicolumn{2}{|c|}{ Incidence, n (\%) } & \multirow[b]{2}{*}{ Crude OR (95\% CI) } & \multirow[b]{2}{*}{ P-value } & \multirow[b]{2}{*}{ Adjusted OR $(95 \% \mathrm{CI})^{\mathrm{a}}$} & \multirow[b]{2}{*}{ P-value } \\
\hline & Gastric cancer & Control & & & & \\
\hline Total & $502(100.00)$ & $502(100.00)$ & & & & \\
\hline \multicolumn{7}{|l|}{ LMP2-60 } \\
\hline Arg/Arg & $338(67.3)$ & $335(66.7)$ & 1 & & 1 & \\
\hline $\mathrm{Arg} / \mathrm{His}$ & $151(30.1)$ & $155(30.9)$ & $0.97(0.74-1.27)$ & 0.799 & $0.97(0.74-1.27)$ & 0.819 \\
\hline His/ His & $13(2.6)$ & $12(2.4)$ & $1.07(0.48-2.39)$ & 0.861 & $1.08(0.48-2.41)$ & 0.857 \\
\hline Arg/His + His/His & $164(32.7)$ & $167(33.3)$ & $0.97(0.75-1.27)$ & 0.840 & $0.98(0.75-1.27)$ & 0.856 \\
\hline \multicolumn{7}{|l|}{ Allelic } \\
\hline Arg & $827(82.4)$ & $825(82.3)$ & $0.99(0.78-1.24)$ & 0.907 & & \\
\hline His & $177(17.6)$ & $179(17.8)$ & & & & \\
\hline HWE & 0.424 & 0.228 & & & & \\
\hline \multicolumn{7}{|l|}{ LMP7-145 } \\
\hline $\mathrm{Gln} / \mathrm{Gln}$ & $310(61.8)$ & 349 (69.5) & 1 & & 1 & \\
\hline Gln/Lys & $169(33.7)$ & $141(28.1)$ & $1.35(1.03-1.77)$ & 0.030 & $1.32(1.00-1.73)$ & 0.049 \\
\hline Lys/Lys & $23(4.6)$ & $12(2.4)$ & $2.16(1.06-4.41)$ & 0.035 & $2.13(1.03-4.39)$ & 0.041 \\
\hline Gln/Lys + Lys/Lys & $192(38.3)$ & $153(30.5)$ & $1.41(1.09-1.84)$ & 0.010 & $1.38(1.06-1.80)$ & 0.017 \\
\hline \multicolumn{7}{|l|}{ Allelic } \\
\hline Gln & 789 (78.6) & 839 (83.6) & $1.39(1.11-1.74)$ & 0.004 & & \\
\hline Lys & $215(21.4)$ & $165(16.4)$ & & & & \\
\hline HWE & 0.995 & 0.613 & & & & \\
\hline
\end{tabular}

${ }^{a}$ Adjusted for age, gender, smoking status, residence, hypertension and diabetes. OR, odds ratio; CI, confidence interval; LMP, low molecular weight protein; HWE, Hardy-Weinberg equilibrium.

the 5 units each of the restriction enzymes $H h a \mathrm{I}$ and $B s m \mathrm{I}$ (New England Biolabs, Ipswich, MA, USA) for $16 \mathrm{~h}$ at $37^{\circ} \mathrm{C}$ and $65^{\circ} \mathrm{C}$, respectively, followed by electrophoresis on a $3 \%$ agarose gel. The Arg/Arg genotype at LMP2-60 was resolved as bands of DNA 79 and 251 bp in length. The His/His genotype yielded a 330-bp band and the Arg/His genotype yielded 79, 251 and 330-bp bands on agarose electrophoresis (Fig. 1A). The Gln/Gln genotype at LMP7-145 yielded 146 and 205-bp bands, the Lys/Lys genotype yielded a 351-bp band and the Gln/Lys genotype produced bands 146, 205 and 351 bp in length on agarose electrophoresis (Fig. 1B). In total, $10 \%$ of the samples were randomly selected and subjected to repeated assays, and the results were all concordant. The DNA direct sequencing method was used to confirm the genotypes of LMP2-60 and LMP7-145 (Fig. 2), and the results were 100\% concordant.

The haplotype frequencies of LMP2 and LMP7 gene polymorphisms were estimated using PHASE2.1 software, based on the Bayesian algorithm (Matthew Stephens Lab, University of Chicago, Chicago, IL, USA) (17).

Statistical analysis. Statistical analysis was performed using SPSS software, version 20.0 (IBM, Armonk, NY, USA). All $\mathrm{P}$-values were two-tailed and $\mathrm{P}<0.05$ was considered to indicate a statistically significant difference. Quantitative variables departing from the normal distribution were summarized as medians and analyzed by the Mann-Whitney U test.
Distributions of categorical variables, and allele and genotype frequencies in cases and controls were compared using the Pearson's $\chi^{2}$ test. The Hardy-Weinberg equilibrium was used to assess the controls using the $\chi^{2}$ goodness of fit test. The odds ratio (OR) and $95 \%$ confidence interval (CI) were used to evaluate the association between polymorphisms or haplotypes and the risk of GC. The crude OR was calculated using the Woolf approximation method and the adjusted OR was computed by multivariate analysis with unconditional logistic regression. The data were adjusted for age, gender, hypertension, diabetes, smoking status and residence.

\section{Results}

Demographic information. The present cohort comprised 502 gastric cancer patients and 502 age and gender-matched cancer-free control individuals. The demographic characteristics of the study subjects are shown in Table I. No significant differences in the distribution of age, gender, hypertension, diabetes and residence were observed between the GC and control groups. However, the proportion of smokers was significantly higher in patients with GC compared with control individuals $(\mathrm{P}=0.003)$.

Polymorphism of LMP2 and LMP7, haplotype analysis and the association with gastric cancer. The distribution of the polymorphisms of LMP2 and LMP7 was within the 
Table III. Associated risk analysis between the gastric cancer and the haplotypes constructed by LMP2/LMP7 gene polymorphisms.

\begin{tabular}{|c|c|c|c|c|c|c|}
\hline \multirow[b]{2}{*}{ Haplotype } & \multicolumn{2}{|c|}{ Incidence, $\mathrm{n}$} & \multirow[b]{2}{*}{ Crude OR (95\% CI) } & \multirow[b]{2}{*}{ P-value } & \multirow[b]{2}{*}{ Adjusted OR $(95 \% \mathrm{CI})^{\mathrm{a}}$} & \multirow[b]{2}{*}{ P-value } \\
\hline & Gastric cancer & Control & & & & \\
\hline LMP2 (Arg)-LMP7 (Gln) & 616 & 659 & 1 & & 1 & \\
\hline LMP2 (Arg)-LMP7 (Lys) & 211 & 165 & $1.37(1.09-1.72)$ & 0.008 & $1.34(1.06-1.70)$ & 0.013 \\
\hline LMP2 (His)-LMP7 (Gln) & 173 & 178 & $1.04(0.82-1.32)$ & 0.746 & $1.04(0.82-1.32)$ & 0.751 \\
\hline LMP2 (His)-LMP7 (Lys) & 4 & 2 & $2.14(0.39-11.72)$ & 0.381 & $2.13(0.38-11.86)$ & 0.387 \\
\hline
\end{tabular}

OR, odds ratio; CI, confidence interval; LMP, low molecular weight protein.
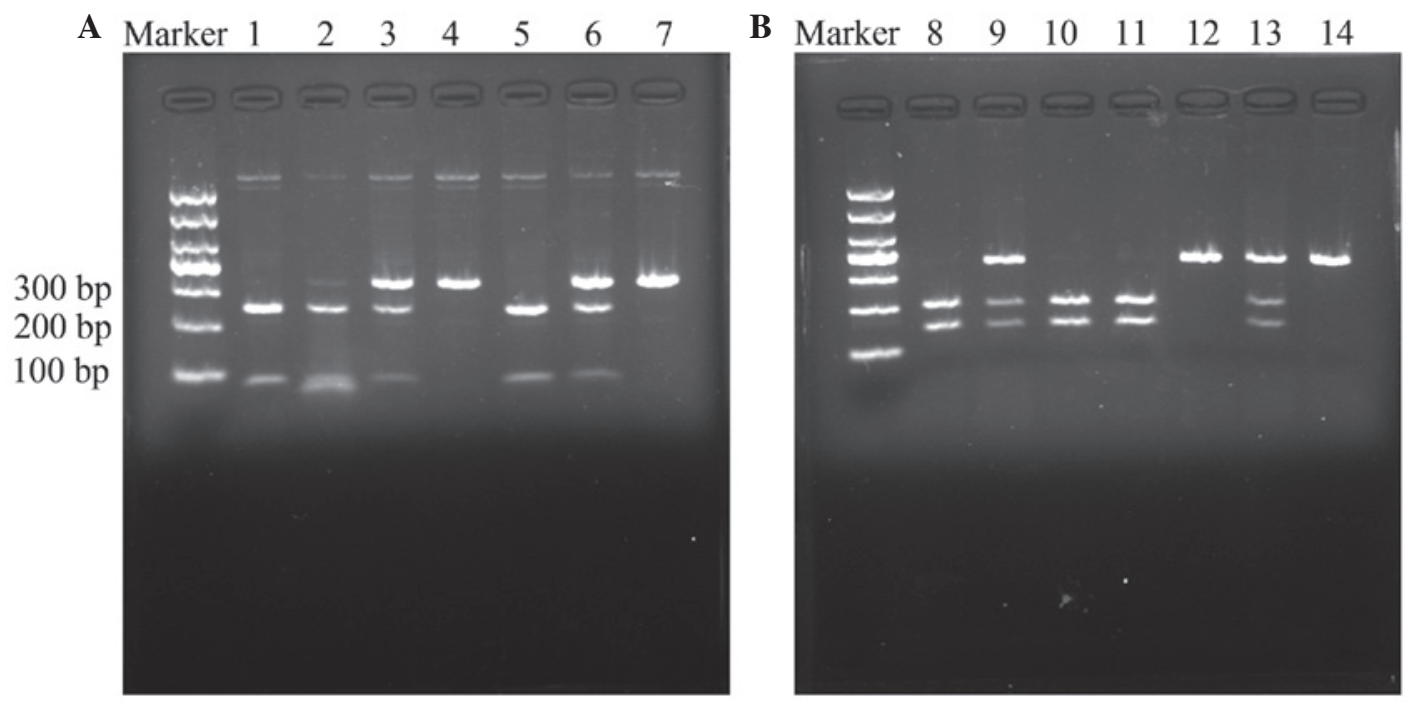

Figure 1. Digestion of polymerase chain reaction products by restriction enzymes. (A) Arg to His genotype variation at codon 60 of LMP2. Lanes 1, 2 and 5 , Arg/Arg homozygous cells, yielding bands of 250 and 79 bp; lanes 3 and 6, Arg/His heterozygous cells, yielding bands of 329, 250 and 79 bp; and lanes 4 and 7 , His/His homozygous cells, yielding a band of 329 bp. (B) Glyn to Lys genotype variation at codon 145 of LMP7. Lanes 8, 10 and 11, Gln/Gln homozygous cells, yielding bands of 205 and 146 bp; lanes 9 and 13, Gln/Lys heterozygous cells, yielding bands of 351, 205 and 146 bp; and lanes 12 and 14, Lys/Lys homozygous cells, yielding a band of $351 \mathrm{bp}$. LMP, low molecular weight protein.

Hardy-Weinberg equilibrium ( $\mathrm{P}>0.05$; Table II). The allele and variant genotypes of the LMP7 gene were significantly different between the patients with GC and control subjects. The frequency of the Lys allele was markedly increased in patients with GC compared with the control subjects $(\mathrm{P}=0.004$; OR, 1.39; 95\% CI, 1.11-1.74). Compared with the Gln/Gln genotype, the frequency of the LMP7-145 Gln/Lys heterozygotic genotype was significantly increased in patients with GC compared with control subjects $(\mathrm{P}=0.049$; adjusted $\mathrm{OR}, 1.32$; 95\% CI, 1.00-1.73). Similarly, the frequency of the Lys/Lys homozygous genotype was increased in GC patients compared with control individuals ( $\mathrm{P}=0.041$; adjusted $\mathrm{OR}, 2.13$; $95 \%$ CI, 1.03-4.39). In addition, with the Gln/Gln genotype as a reference, the variant genotypes (Gln/Lys + Lys/Lys) were associated with an increased susceptibility to $\mathrm{GC}(\mathrm{P}=0.017$; adjusted OR, 1.38; 95\% CI, 1.06-1.80) subsequent to adjustment of the data for age, gender, smoking status, hypertension, diabetes and residence. However, no significant association between the LMP2-60 polymorphism and GC was observed in the present study $(\mathrm{P}>0.05$; Table II).

Each individual haplotype derived from polymorphism of the LMP2 and LMP7 genes was constructed using
PHASE2.1 software based on the Bayesian algorithm. When compared with the most frequent Arg-Gln haplotype, the Arg-Lys haplotype was associated with the risk of GC ( $\mathrm{P}=0.013$; adjusted OR, 1.34; 95\% CI, 1.06-1.70). However, no association was detected between the other two haplotypes and the risk of GC (Table III).

Stratified analysis of polymorphisms and gastric cancer risk. The results of the stratified analysis of the LMP2 and LMP7 polymorphisms according to the median age of controls (59 years), gender, smoking status and residence are summarized in Table IV. The variant genotypes of LMP7-145, consisting of the Gln/Lys and Lys/Lys variants, significantly increased the risk of GC in subjects aged $>59$ years $(\mathrm{P}=0.024$; adjusted OR, 1.57; 95\% CI, 1.06-2.31), males ( $\mathrm{P}=0.015$; adjusted OR, 1.48; 95\% CI, 1.08-2.03) and non-smokers ( $\mathrm{P}=0.010$; adjusted OR, 1.48; 95\% CI, 1.10-1.99). However, the association was not statistically significant in subjects aged $\leq 59$, females and smokers. No statistically significant association was identified between the location of residence and the presence of polymorphisms or the susceptibility to GC. Furthermore, no significant association was observed between 


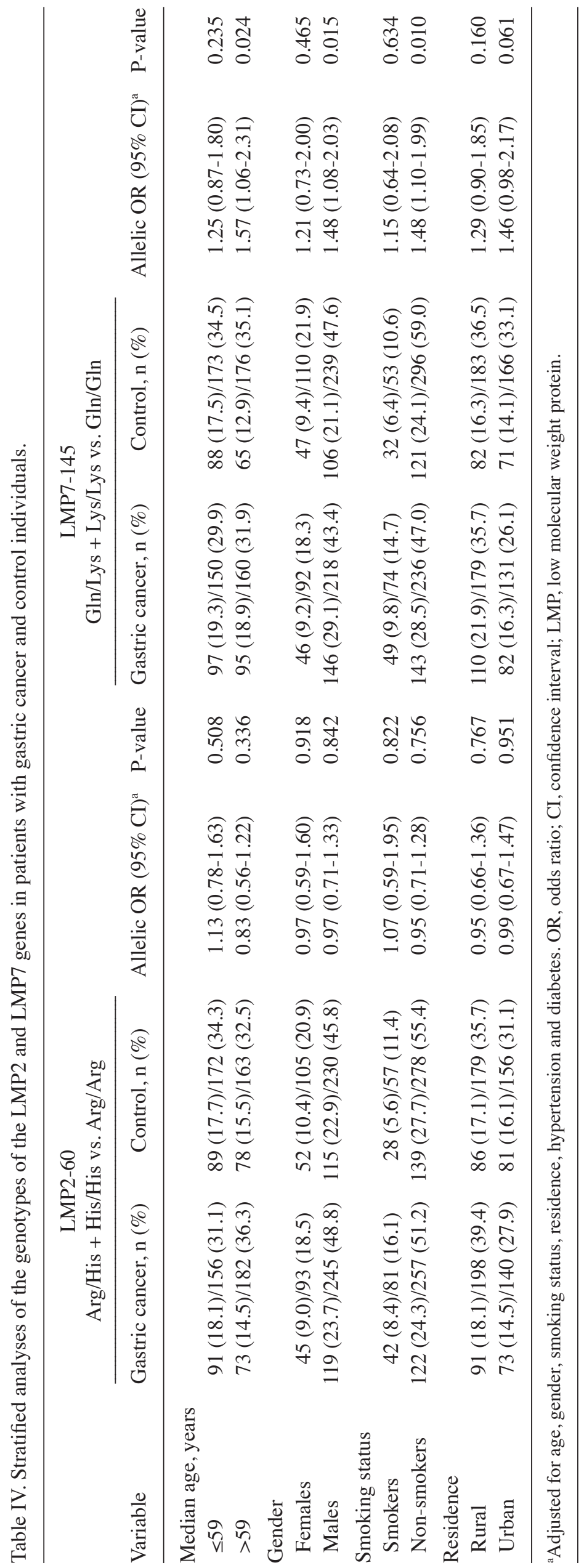




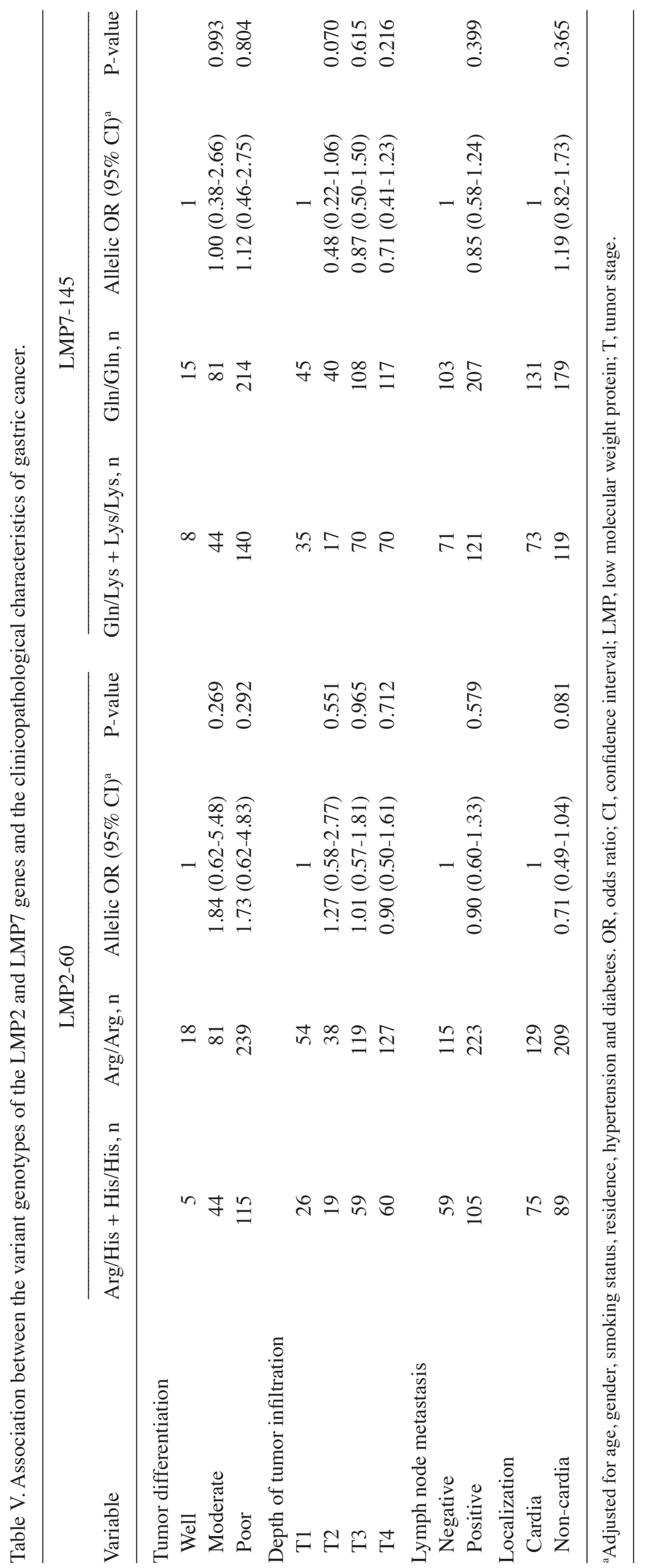


A

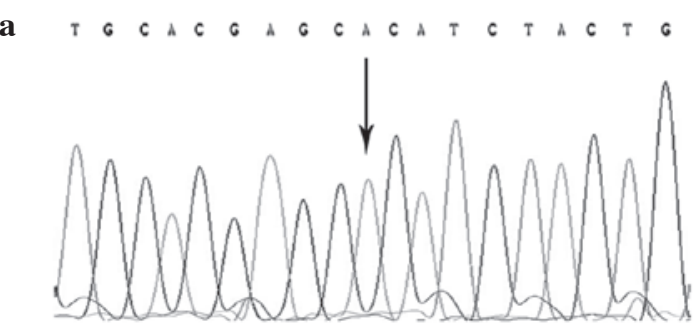

b

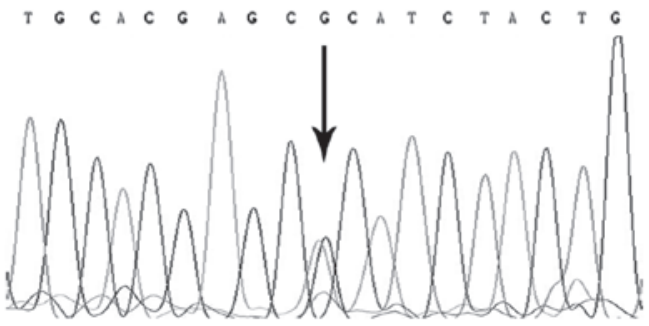

c

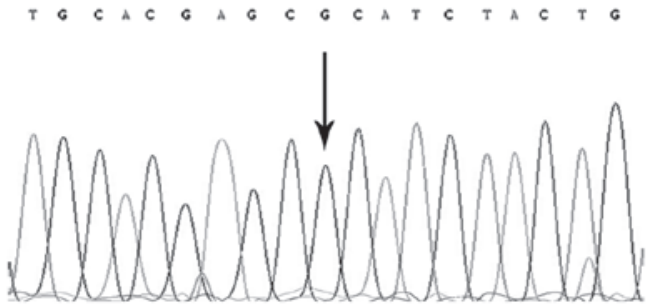

B

a

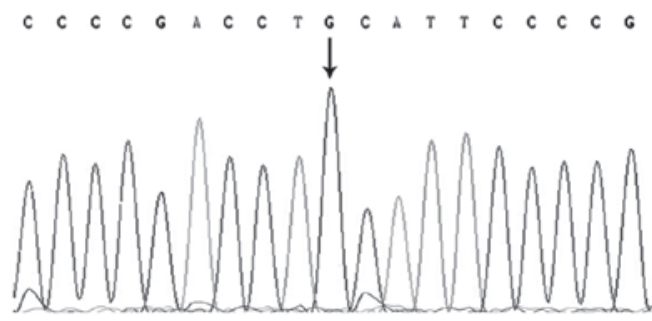

b
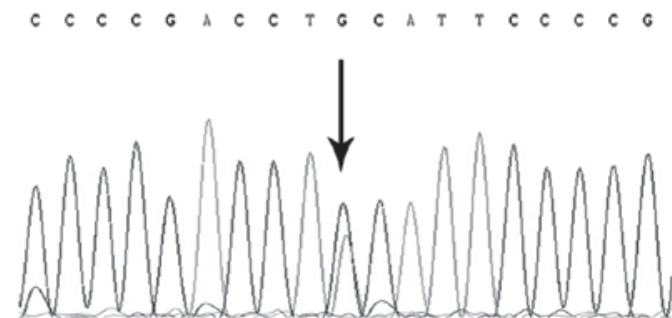

c

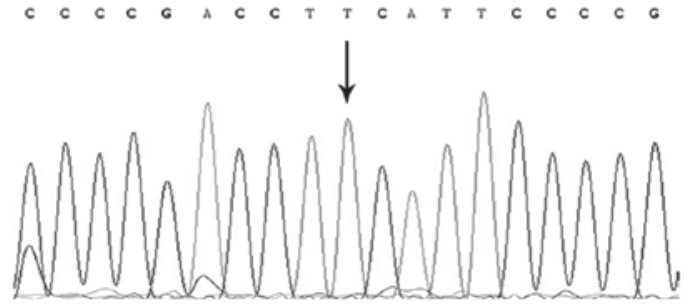

Figure 2. Direct sequencing results for the polymorphisms of the LMP2 and LMP7 genes. The polymorphisms were detected by restriction fragment length polymorphism assay and confirmed by direct polymerase chain reaction sequencing. The single base indicated by a black arrowhead is the site of the small nucleotide polymorphism. (A) Representative DNA sequencing results of the (a) Arg/Arg, (b) Arg/His and (c) His/His genotypes of the Arg to His mutation at codon 60 of the LMP2 gene. (B) Representative DNA sequencing results of the (a) Gln/Gln, (b) Gln/Lys and (c) Lys/Lys genotypes of the Gln to Lys mutation at codon 145 of the LMP7 gene. LMP, low molecular weight protein. the LMP2-60 polymorphisms and age, gender, smoking status and residence.

The effects of the LMP2 and LMP7 polymorphisms were evaluated subsequent to stratification of the GC patients by clinicopathological characteristics (Table V), which revealed no significant association between the SNPs and the depth of tumor infiltration, grade of differentiation, lymph node metastasis or location of the primary cancer.

\section{Discussion}

Genetic and environmental factors influence the type of malignant tumor that develops and the growth of the tumor (18). In the present study, the association between LMP2 and LMP7 polymorphisms and the risk of GC was investigated. The LMP2 and LMP7 genes are located in a short, 800-kb region of the short arm of chromosome 6, which encodes the proteins essential for sequential peptide processing in the pathway of major histocompatibility complex (MHC)-I antigen presentation $(19,20)$.

Cancer cells escape immune recognition through reduced MHC expression, as the presentation of immunogenic tumor peptides by HLA class I antigens is a prerequisite for a successful antitumor immune response (21). The efficient expression of peptide-HLA complexes at the cell surface depends on the type and quantity of the peptides that are produced and processed (14). Overexpression of the LMP2 and LMP7 gene may lead to the production of an insufficient peptide level, which may allow cancer cells to escape immune processing, leading to GC development. It was hypothesized that the Arg to His polymorphism at LMP2-60 and Gln to Lys polymorphism at LMP7-145 may lead to functional alterations that affect the development and progression of gastric cancer.

To verify the hypothesis that LMP2 and LMP7 polymorphisms may be associated with the risk of GC, a hospital-based case-control study that consisted of 502 gastric cancer patients and 502 healthy control individuals was performed. For LMP7, the data revealed that the frequency of the Lys/Lys genotype at LMP7-145 was significantly increased in gastric cancer patients compared with control individuals $(\mathrm{P}=0.041$; adjusted OR, 2.13; 95\% CI, 1.03-4.39). However, the Gln/Lys genotype was more common in patients with GC compared with control individuals ( $\mathrm{P}=0.049$; adjusted $\mathrm{OR}, 1.32$; 95\% CI, 1.00-1.73). Compared with the common genotype Gln/Gln, patients possessing the Gln/Lys + Lys/Lys genotype demonstrated a $38 \%$ increased risk of developing $\mathrm{GC}(\mathrm{P}=0.017$; adjusted $\mathrm{OR}$, 1.38; 95\% CI, 1.06-1.80). Separate analysis of the Lys allele revealed that this allele was more prevalent in patients with GC compared with control individuals (21.4 vs. $16.4 \%$; $\mathrm{P}=0.004$; OR, 1.39; 95\% CI, 1.11-1.74). These results indicated that the Gln/Lys + Lys/Lys genotypes at LMP7-145, in which Gln is substituted with Lys, were significantly associated with an increased risk of GC and the Lys allele may be an independent risk factor of GC.

No association was identified between the LMP2-60 genotypes or alleles and the risk of GC. These results were consistent with those reported in the majority of previous studies that investigated the association between polymorphisms in LMP2 and LMP7, and other diseases $(11-13,22,23)$. However, Ozbas-Gerceker et al reported that LMP2 plays a 
role in the development of acute myeloid leukemia and multiple myeloma, whereas LMP7 is not a risk factor for hematological malignancies (24). The discrepancy between these results and the present findings may be attributed to the fact that the same polymorphism may exert different genetic effects on various types of cancer $(25,26)$.

Overall, the present statistical analysis revealed a significant association between the risk of GC and polymorphisms of LMP7, but not polymorphisms of LMP2, and these findings are in agreement with the LMP2/7 gene expression levels in human GC cells. According to Kang et al, MHC class I surface expression is associated, in the majority of cases, with the expression of the LMP7 gene, regardless of the LMP2, TAP1, TAP2 or MHC class I genes (27).

Based on the two polymorphisms of LMP2 and LMP7 being located close to each other in the MHC class II region, four haplotypes were constructed. The present results indicated that compared with the most frequent Arg-Gln haplotype, the Arg-Lys haplotype was more frequent in patients with GC compared with control individuals $(\mathrm{P}=0.013$; adjusted $\mathrm{OR}, 1.34$; 95\% CI, 1.06-1.70). This was consistent with the effects of the single LMP7 SNP. However, the OR was low compared with the OR of the Lys allele of the LMP7 polymorphism alone. In addition, no association was identified between the incidence of the His-Lys haplotype and the occurrence if GC. These results suggest that the LMP2 polymorphism may weaken the susceptibility of the haplotype to GC. However, further fine mapping studies are required to clarify this mechanism.

In the stratified analysis of the present study, the data revealed that polymorphisms at LMP7-145 were associated with an increased risk of GC in patients aged $>59$ years, but not in those aged $\leq 59$ years. Carcinogenesis is an accumulation of genetic events during aging, and the age-dependent increase in the incidence of GC demonstrates a steep slope (28). The increased risk of GC in the elderly indicates that gene-environment interactions may be involved in carcinogenesis, and the LMP7-145 genotype effects tended to be age-specific. A significantly elevated risk was also identified in male subjects. A previous study performed using a Chinese cohort reported that non-cardia gastric cancer is more common in males than females, with a ratio of $\sim 2: 1$, and that gastric cardia cancer has an increased male to female ratio of 4.1:1 (29). These findings suggest that LMP7-145 polymorphisms play an important role in men with GC. In the present stratified analysis of smoking status, an evident association between polymorphisms at LMP7-145 and the risk of GC was observed in non-smokers, but not in smokers. Tobacco smoke is a known independent risk factor for GC (3). The association between the incidence of polymorphism and GC risk may be masked by the overwhelming accumulated exposure to tobacco carcinogens in smokers, resulting in a more significant association among non-smokers (30). However, additional studies are required to clarify the association between the LMP7-145 polymorphism and GC in men, non-smokers and elderly patients.

The present study demonstrates several limitations. Firstly, the relatively small sample size may have limited the statistical power of the data. Secondly, the consecutive enrollment of subjects from the same hospital and during the same period may have led to a selection bias. However, the genotype distribution in the healthy control individuals in the present study was compatible with the Hardy-Weinberg expectations. Thirdly,
Helicobacter pylori is an independent risk factor for gastric cancer, but the variable was not tested for, as it was unethical to perform the test for Helicobacter pylori on each subject, particularly in the control individuals. Additionally, in the stratified analysis, the risk association with Lauren's classifications (31) and the consumption of alcohol was not evaluated. Future studies should include these variables in the stratified analysis. Lastly, due to the analysis being limited to individuals from the Chinese population, extrapolation of the present results to other regions and ethnicities should be performed with caution.

In conclusion, to the best of our knowledge, the present study is the first to demonstrate that LMP7 polymorphisms are associated with an increased risk of GC in a Chinese population, particularly in older individuals, non-smokers and males. Additional studies with larger sample sizes and the inclusion of various populations, as well as functional studies, are required to verify the present initial findings.

\section{Acknowledgements}

This study was supported by grants from the Medical Zhong Dian Ren Cai Project of Jiangsu Province (grant no., RC2011059), Natural Science Foundation of Jiangsu Province [grant no., BK20131447 (DA13)], Six RenCai Gaofeng, 333 Project Leader of Jiangsu Province to L. Yang and A Project Funded by the Priority Academic Program Development of Jiangsu Higher Education Institutions (PAPD) (grant no., JX10231801).

\section{References}

1. Danaei G, Vander Hoorn S, Lopez AD, Murray CJ and Ezzati M; Comparative Risk Assessment collaborating group (Cancers): Causes of cancer in the world: Comparative risk assessment of nine behavioural and environmental risk factors. Lancet 366: 1784-1793, 2005

2. Pisani P, Parkin DM, Bray F and Ferlay J: Erratum: Estimates of the worldwide mortality from 25 cancers in 1990. Int. J. Cancer, 83, 18-29 (1999). Int J Cancer 83: 870-873, 1999.

3. Parkin DM: International variation. Oncogene 23: 6329-6340, 2004.

4. Wu MS, Chen CJ and Lin JT: Host-environment interactions: Their impact on progression from gastric inflammation to carcinogenesis and on development of new approaches to prevent and treat gastric cancer. Cancer Epidemiol Biomarkers Prev 14: 1878-1882, 2005.

5. Zhu H, Yang L, Zhou B, Yu R, Tang N and Wang B: Myeloperoxidase G-463A polymorphism and the risk of gastric cancer: A case-control study. Carcinogenesis 27: 2491-2496, 2006.

6. Sun Q, Gu H, Zeng Y, Xia Y, Wang Y, Jing Y, Yang L and Wang B: Hsa-mir-27a genetic variant contributes to gastric cancer susceptibility through affecting miR-27a and target gene expression. Cancer Sci 101: 2241-2247, 2010.

7. Yang L, Gu HJ, Zhu HJ, Sun QM, Cong RH, Zhou B, Tang NP and Wang B: Tissue inhibitor of metalloproteinase-2 G-418C polymorphism is associated with an increased risk of gastric cancer in a Chinese population. Eur J Surg Oncol 34: 636-641, 2008.

8. Yang L, Zhu H, Zhou B, Gu H, Yan H, Tang N, Dong H, Sun Q, Cong $\mathrm{R}$, Chen $\mathrm{G}$, et al: The association between the survivin $\mathrm{C}-31 \mathrm{G}$ polymorphism and gastric cancer risk in a Chinese population. Dig Dis Sci 54: 1021-1028, 2009.

9. Suzuki K, Sahara H, Okada Y, Yasoshima T, Hirohashi Y, Nabeta Y, Hirai I, Torigoe T, Takahashi S, Matsuura A, et al: Identification of natural antigenic peptides of a human gastric signet ring cell carcinoma recognized by HLA-A31-restricted cytotoxic T lymphocytes. J Immunol 163: 2783-2791, 1999.

10. Ishigami S, Natsugoe S, Nakajo A, Arigami T, Kitazono M, Okumura H, Matsumoto M, Uchikado Y, Setoyama T, et al: HLA-class I expression in gastric cancer. J Surg Oncol 97: 605-608, 2008. 
11. Ferris RL, Hunt JL and Ferrone S: Human leukocyte antigen (HLA) class I defects in head and neck cancer: Molecular mechanisms and clinical significance. Immunol Res 33: 113-133, 2005.

12. Grommé $\mathrm{M}$ and Neefjes J: Antigen degradation or presentation by MHC class I molecules via classical and non-classical pathways. Mol Immunol 39: 181-202, 2002.

13. Song L, Ma N, Han L, Yan H, Yan B, Yuan Z and Cao B: Association between LMP2/LMP7 genetic variability and the metastasis risk of ovarian cancer in Chinese women in Beijing. Hum Immunol 75: 239-244, 2014.

14. Fellerhoff B, Gu S, Laumbacher B, Nerlich AG, Weiss EH, Glas J, Kopp R, Johnson JP and Wank R: The LMP7-K allele of the immunoproteasome exhibits reduced transcript stability and predicts high risk of colon cancer. Cancer Res 71: 7145-7154, 2011.

15. Cao B, Tian X, Li Y, Jiang P, Ning T, Xing H, Zhao Y, Zhang C, Shi X, Chen D, et al: LMP7/TAP2 gene polymorphisms and HPV infection in esophageal carcinoma patients from a high incidence area in China. Carcinogenesis 26: 1280-1284, 2005.

16. Zhu H, Yang L, Zhou B, Yu R, Tang N and Wang B: Myeloperoxidase G-463A polymorphism and the risk of gastric cancer: A case-control study. Carcinogenesis 27: 2491-2496, 2006.

17. Stephens M and Donnelly P: A comparison of bayesian methods for haplotype reconstruction from population genotype data. Am J Hum Genet 73: 1162-1169, 2003.

18. Hemminki K, Försti A and Bermejo JL: Gene-environment interactions in cancer: do they exist? Ann NY Acad Sci 1076: 137-148, 2006.

19. Basler M, Kirk CJ and Groettrup M: The immunoproteasome in antigen processing and other immunological functions. Curr Opin Immunol 25: 74-80, 2013.

20. Hussong SA, Roehrich H, Kapphahn RJ, Maldonado M, Pardue MT and Ferrington DA: A novel role for the immunoproteasome in retinal function. Invest Ophthalmol Vis Sci 52: 714-723, 2011

21. Lee YM, Leu SY, Chiang H,Fung CP and Liu WT: Human papillomavirus type 18 in colorectal cancer. J Microbiol Immunol Infect 34: 87-91, 2001.
22. Lv Y, Yan B, Yang H, Liu J, Zhong W, Li K, Chen Z and Xu C: LMP2/LMP7 gene variant: A risk factor for intestinal Mycobacterium tuberculosis infection in the Chinese population. J Gastroenterol Hepatol 26: 1145-1150, 2011.

23. Camarena A, Aquino-Galvez A, Falfán-Valencia R, Sánchez G, Montaño M, Ramos C, Juárez A, García-de-Alba C, Granados J and Selman M: PSMB8 (LMP7) but not PSMB9 (LMP2) gene polymorphisms are associated to pigeon breeder's hypersensitivity pneumonitis. Respir Med 104: 889-894, 2010.

24. Ozbas-Gerceker F, Bozman N, Kok S, Pehlivan M, Yilmaz M, Pehlivan S and Oguzkan-Balci S: Association of an LMP2 polymorphism with acute myeloid leukemia and multiple myeloma. Asian Pac J Cancer Prev 14: 6399-6402, 2013.

25. Yu Z, Liu Q, Huang C, Wu M and Li G: The interleukin 10 $-819 \mathrm{C} / \mathrm{T}$ polymorphism and cancer risk: A HuGE review and meta-analysis of 73 studies including 15,942 cases and 22,336 controls. OMICS 17: 200-214, 2013.

26. Zhang YM, Zhou XC, Xu Z and Tang CJ: Meta-analysis of epidemiological studies of association of two polymorphisms in the interleukin-10 gene promoter and colorectal cancer risk. Genet Mol Res 11: 3389-3397, 2012.

27. Kang JK, Yoon SJ, Kim NK and Heo DS: The expression of MHC class I, TAP1/2, and LMP2/7 gene in human gastric cancer cell lines. Int J Oncol 16: 1159-1163, 2000.

28. Milne AN, Carvalho R, Morsink FM, Musler AR, de Leng WW, Ristimäki A and Offerhaus GJ: Early-onset gastric cancers have a different molecular expression profile than conventional gastric cancers. Mod Pathol 19: 564-572, 2006.

29. Kelley JR and Duggan JM: Gastric cancer epidemiology and risk factors. J Clin Epidemiol 56: 1-9, 2003.

30. Gu H, Yang L, Sun Q, Zhou B, Tang N, Cong R, Zeng Y and Wang B: Gly82Ser polymorphism of the receptor for advanced glycation end products is associated with an increased risk of gastric cancer in a Chinese population. Clin Cancer Res 14: 3627-3632, 2008.

31. Lauren P: The two histological main types of gastric carcinoma: Diffuse and so-called intestinal-type carcinoma. An attempt at a histo-clinical classification. Acta Pathol Microbiol Scand 64: 31-49, 1965. 